\title{
Road Network Extraction from High Resolution Multispectral Satellite Imagery Based on Object Oriented Techniques
}

\author{
M. Kumar ${ }^{\text {a, }}{ }^{*}$ R.K.Singh ${ }^{\text {b }}$, P.L.N.Raju ${ }^{\text {a }}$, Y.V.N.Krishnamurthy ${ }^{\text {a }}$ \\ a Indian Institute of Remote Sensing, Dehradun, India- (minakshi ,raju, yvnk)@iirs.gov.in \\ ${ }^{\mathrm{b}}$ Uttarakhand Technical University, Dehradun , India - (rksinghkec12@ rediffmail.com)
}

\section{Commission VIII WG VIII/8}

KEY WORDS: High Resolution Multispectral data, Multi-resolution Segmentation, Road Extraction, Fuzzy Classification Image Fusion.

\begin{abstract}
:
High Resolution satellite Imagery is an important source for road network extraction for urban road database creation, refinement and updating. However due to complexity of the scene in an urban environment, automated extraction of such features using various line and edge detection algorithms is limited. In this paper we present an integrated approach to extract road network from high resolution space imagery. The proposed approach begins with segmentation of the scene with Multi-resolution Object Oriented segmentation. This step focuses on exploiting both spatial and spectral information for the target feature extraction. The road regions are automatically identified using a soft fuzzy classifier based on a set of predefined membership functions. A number of shape descriptors are computed to reduce the misclassifications between road and other spectrally similar objects. The detected road segments are further refined using morphological operations to form final road network, which is then evaluated for its completeness, correctness and quality. The experiments were carried out of fused IKONOS 2, Quick bird ,Worldview 2 Products with fused resolution's ranging from $0.5 \mathrm{~m}$ to $1 \mathrm{~m}$. Results indicate that the proposed methodology is effective in extracting accurate road networks from high resolution imagery.
\end{abstract}

\section{INTRODUCTION}

The availability of space borne images at finer spatial resolutions has opened new vistas in the areas of detailed automated feature extraction. Mapping and extraction of features from satellite imagery is a fundamental operation for updating maps. While road extraction has been performed by digitizing maps, the update and refinement of the road geometry is often based on aerial imagery or high resolution satellite imagery. Automatic road extraction from Remote sensing imagery has attracted much attention for the last few decades. Automatic feature extraction has become a main objective to save time in updating data, and as a result of this, people are changing progressively to new procedures (Kumar2009 a, Jin , 2005. Automated road information extraction from highresolution images holds great potential for significant reduction of database development cost and turnaround time and vigorous efforts have been made to develop better algorithms and methodologies for this purpose (Mokhtarzade 2008, Mena, 2005).

The most common automated techniques for road extraction in images with low resolution are detection and following of lines. However automatic road extraction from dense urban areas using very high spatial resolution images is a challenging issue due to the high complex image scene and occlusions caused by high buildings and their shadows. Nevertheless some attempts have been made in this area and have achieved abundant results. Mohammadzadeh, A. et. al (2008) carried out an approach to detect road network from high resolution image using combination of a developed fuzzy system and mathematical morphology. This method was successfully executed on different regions including urban, suburban and rural areas. In Mena, J.B et al (2005), a method for automatic road extraction in rural and semi-urban areas was presented. The system includes four different modules: data pre-processing; binary segmentation based on three levels of texture statistical evaluation; automatic vectorization by means of skeletal extraction; and finally a module for system evaluation. Wang, Y et al (2008) presented an approach of multi-resolution analysis to extract road information from high resolution imagery. In this method, first, high resolution imagery was divided into a series of different resolution images. According to the representation characteristics of roads at different resolution, roads geometrical, radiometric, and topological attributes were analysed and carried out a variety of processes such as image enhancement, edge detection, road finding, tracking and linking.

Inspite of all the efforts existing road extraction methods still have limitations in popularization and application. Further the extraction accuracy cannot satisfy the needs of engineering application. The automation is in a relatively low level and the performance is limited by either road materials or complex road networks or the obstructions causing the visibility of road in a satellite image. Full automation of the extraction of topographic objects is currently practically impossible for almost all applications and thus a combination with human interaction is necessary. With an aim to exploit the high content of textural, spatial and spectral information present in high resolution satellite imagery an attempt has been made to develop a methodology to extract roads and evaluate the road extractions

* Corresponding author. This is useful to know for communication with the appropriate person in cases with more than one author. 
in an urban environment utilizing the recent advanced techniques at a low cost.

\section{DATA AND RESEARCH METHODS}

The experiment has been carried out on different kind of road segment ranging from planned and unplanned urban road segments as well as rural roads. The satellite data utilized for this study is acquired by Worldview2, Quick Bird, IKONOS and high-resolution commercial earth observation satellites collecting data in panchromatic mode to $0.4,0.61 \mathrm{~m}$ and $1 \mathrm{~m}$ respectively, pixel resolution degree of detail. The satellite collects panchromatic (black \& white) imagery as well as multispectral imagery at 1.6, 2.5 and 4 meter resolution.

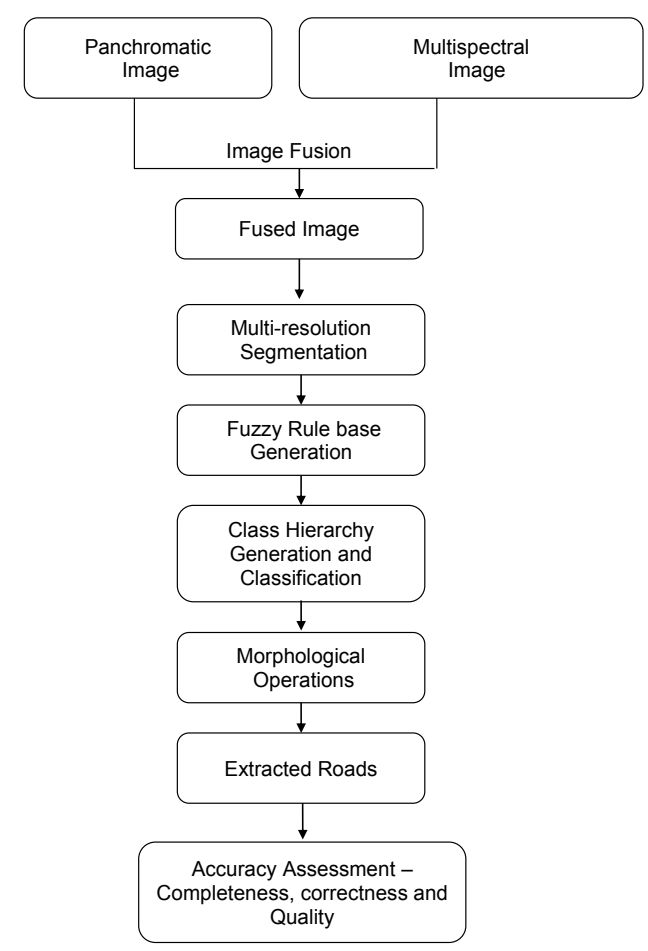

Figure 1 : Integrated Work Flow

Figure 1 illustrates the workflow of the integrated processing for road feature extraction from urban environment. The prerequisite to road extraction is the pre-processing of raw images which include registration of the image, merging and selection of an area of interest. Image registration is the process of transforming the different sets of data into one coordinate system. Registration is necessary in order to be able to compare or integrate the data obtained from different measurements. The panchromatic and multispectral images were registered with the same coordinate system to a sub pixel accuracy. Fusion is the process of integrating two or more images of same or different resolutions using certain approach to acquire a composite image with high degree of information. The two registered images were fused together with principal component analysis technique as all the four multispectral bands could be used, as compared to other techniques where fusion is limited to three bands.

As the resolution of the fused product was large road with both the bounding edges were visible on the image. The process of detecting objects in an image consists of a sequence of segmentation and classification procedures. Multi-resolution
Segmentation was carried out by applying different scale parameter, shape factor, smoothness and compactness. The scale determines the size of an image object formed during segmentation. It measures the maximum change in heterogeneity that may occur when merging of two image objects. The heterogeneity criterion then comprises of the colour / tone and shape. This process works on the spectral behaviour of the pixels. The spectral criterion is the change in heterogeneity. The shape criterion is composed of smoothness and compactness of the image object. Smoothness criteria smoothens the borders of objects while compactness analyses how compactly the images are bounded together. Once suitable parameters are identified, the pixels having a homogeneous pattern are grouped into image objects. Different image objects have different spectral, shape, and hierarchical characteristics. A rule set then has to be defined which is needed to separate different classes. For the study area, the image was segmented in 6 different levels with scale parameters ranging from 10 with an increment of 5 with a constant shape factor of 0.3 , compactness and smoothness of 0.1 and 0.9 respectively. It was observed that the road objects were well defined at Level 5 with a scale parameter of 30 which was used for further image analysis.

While developing a rule set information about the attributes of image objects, such as their color layer value, texture or shape is needed. Layer values evaluate the mean and standard deviation of an image object's pixel value and the object's relations to other image object's pixel values. Shape features evaluate the image object's shape in a variety of respects. If image objects of a certain class stand out because of their shape, a rule that describes them can be formed. In addition a large number of features based upon the co-occurrence matrix after Haralick can be utilized. Therefore rules are defined using fuzzy membership functions and Image object attribute values. The fuzzy rules applicable for road side extraction on the test site are tabulated in table 1 . In the next step a class hierarchy of road class was generated, which contains set of object-based rules with their corresponding membership values. Once these rules are collectively compiled in a class hierarchy, the classification of road can be done. The output classified image was further processed for the purpose of smoothening with a close function morphological operation using $5 \times 5$ kernel. Figure 2 illustrates the results of the steps of processing. Figure 3 a presents major road segment and $3 \mathrm{~b}$ presents the complex road segments overlaid on the satellite imagery.
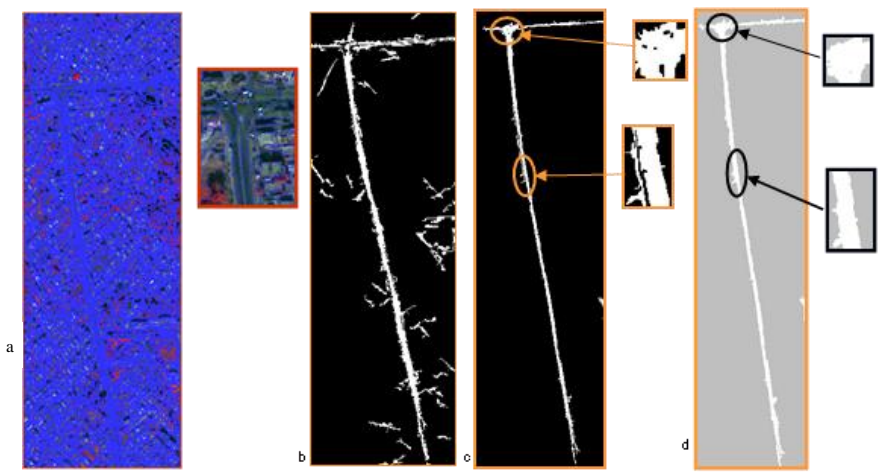

Figure 2 (a) Segmented image (b) Road Class (c) Refined road class (d) Final road extracted Insets: Areas needing attention 
Table 1 Image Object Parameters with Membership Functions

\begin{tabular}{|l|l|l|l|}
\hline $\begin{array}{l}\text { Sl. } \\
\text { No }\end{array}$ & Feature/Parameter & $\begin{array}{l}\text { Membership } \\
\text { Functions }\end{array}$ & $\begin{array}{l}\text { Membership } \\
\text { Values }\end{array}$ \\
\hline 1 & $\begin{array}{l}\text { Avg. length of } \\
\text { edges (polygon) }\end{array}$ & About range & $3.5-33$ \\
\hline 2 & Border Length & Boolean & $100-238$ \\
\hline 3 & Compactness & Full range & $-0.0439-0.1661$ \\
\hline 4 & Density & Full range & $0.2-1.6$ \\
\hline 5 & Length & Boolean & $34-53$ \\
\hline 6 & $\begin{array}{l}\text { Length of main } \\
\text { line }\end{array}$ & Full range & $50-890$ \\
\hline 7 & Length/width ratio & Full range & $2.16-57$ \\
\hline 8 & $\begin{array}{l}\text { Number of } \\
\text { branches of length }\end{array}$ & Full range & $2-25$ \\
\hline 9 & Shape Index & Larger than & $-1-5.5$ \\
\hline 10 & $\begin{array}{l}\text { Width (only main } \\
\text { line) }\end{array}$ & About range & $4-19$ \\
\hline 11 & Asymmetry & Full range & $0.6832-0.6833$ \\
\hline 12 & Elliptic Fit & Full range & $0.3424-0.34244$ \\
\hline 13 & $\begin{array}{l}\text { Std. dev of length } \\
\text { of edges }\end{array}$ & Larger than & $30.69-42.62$ \\
\hline
\end{tabular}
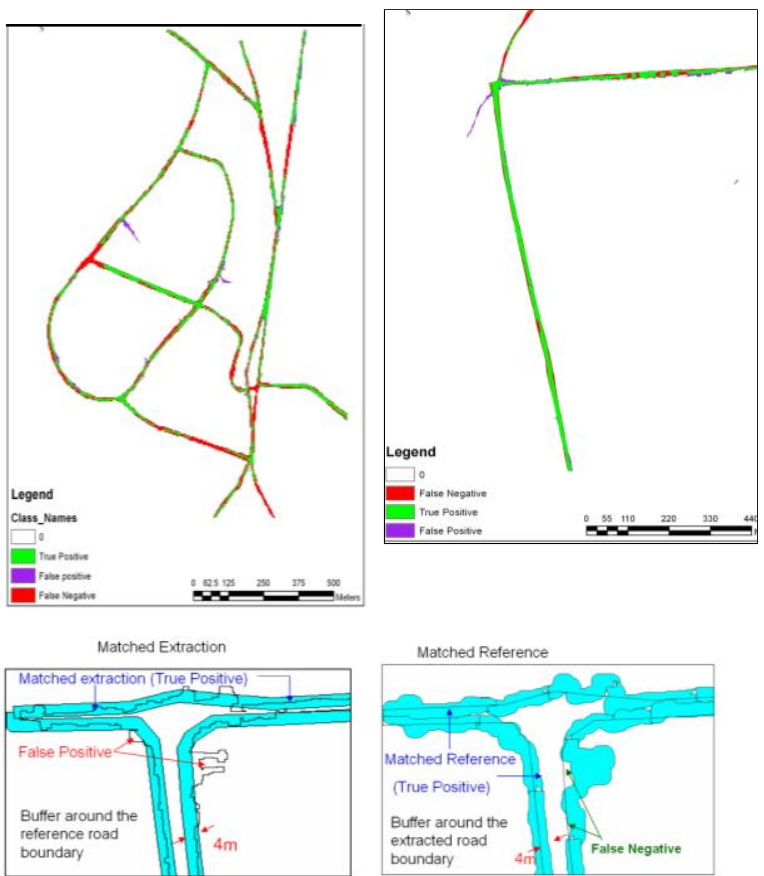

Figure 4 : Road extraction evaluation

In the second step, matching was performed in the other direction. The buffer was now constructed around the extracted road data, and the parts of the reference data lying in the buffer are considered to be matched. In case of low redundancy, their length can be approximated with TP. The unmatched reference data are denoted as false negative with length FN. Figure 4 illustrates presents the matched roads as well as the unmatched portions.

The completeness is the percentage of the reference data which is explained by the extracted data, i.e., the percentage of the reference network which lies within the buffer around the extracted data. The correctness represents the percentage of correctly extracted road data, i.e., the percentage of the extracted data which lie within the buffer around the network. The quality is a measure of the final result of combining completeness and correctness into a single measure. The overall quality achieved was $72.36 \%$, completeness and correctness were $87.98 \%$ and $79.90 \%$ respectively.

\section{CONCLUSION} plotted road axis and boundaries in the reference data. The matching accuracy of extracted road primitives to the reference network is evaluated and the quality measures of correctness, completeness based on the above matching process were quantified.

Matching was performed according to the buffer method and in the first step; a buffer of constant pre-defined width (buffer width) was constructed around the reference road data. The buffer width used was $1 / 3$ of road width. The parts of the extracted data within the buffer are considered as matched. Following the notation of (Shufelt J.A, 1994) the matched extracted data denoted as true positive with length TP emphasizing the fact that the extraction algorithm has indeed found a road, the unmatched extracted data is denoted as false positive with length FP, because the extracted road hypotheses are incorrect.
In this paper, an approach to detect road from high resolution image using was carried out. The approach used in this research was focused on extracting the complete road boundary from the fused high spatial and multispectral image. Upon the analysis area contains structures like trees and vehicles that make the road extraction process bit more difficult. The obstacles on road which make the road boundary difficult are the tree line bounding the road segments, shadows of high buildings fall on the road and the tone of the road spectrally mixing up to with building rooftops. Another problem encountered was different structures that form main road. Some road segments are made up of a combination of metalled road and concrete road, where as some segments of main road composed of metalled and nonmetalled roads and other areas have 'tracks' along the road side. using the method described above, it was found that the study 
Among them, the extracted road boundary comprised the metalled road. . Moreover high resolution imagery reveals detailed information about ground flatness. This utilized detailed information causes imprecise road extraction using pixel-based approaches due to high tonal variation.

Although major road boundary has been extracted, the minor roads are still left to be extracted. Initially, attempt had been made to extract major road as well as minor road simultaneously, but the result obtained gave an indication that for minor road, segmentation parameter chosen should be smaller as compared a large segment which satisfies for extraction of main road. Hence extraction of the remaining minor roads was carried out as a separate study. The proposed approach is well suited for WorldView IKONOS and Quickbird images with a resolution of $(0.4 \mathrm{~m})$ to $(2.5 \mathrm{~m})$. However the algorithm and parameters behaviour expected to be similar on other available resolution like IRS- CARTOSAT high resolution data which can be further investigated

\section{REFERENCES}

Bouziani, M K. Goita and D-C. He, "Rule-based classification of a very high resolution image in an urban environment using multispectral segmentation guided by cartographic data," IEEE Transactions. Geoscience Remote Sensing. vol. 48, no. 8, pp. 3198-3211, Aug. 2010

Chen C., H., Pau L. F., Wang P. S. P. The Handbook of Pattern Recognition and Computer Vision (2nd Edition), by (eds.), pp. 207-248, World Scientific Publishing Co., 1998. Chapter 2.1 Texture Analysis by Mihran Tuceryan and Anil K. Jain

Heipke C., Mayer H., Wiedemann C. 1997. Evaluation of Automatic Road Extraction. International Archives of Photogrammetry and Remote Sensing, Vol. 32, Part 3-4W2.

Jin X, Davis C. H. 2005. An Integrated System for Automatic Road Mapping from High-resolution Multi-spectral Satellite Imagery by Information Fusion. Elsevier B. V, pp. 257-273. Doi : 10.1016/j.inffus.004.06.003.

Gonzalez R.C, Woods R.E \& Eddins S.L 2010. Digital Image Processing Using Matlab 2Edition. Tata Mc Graw Hill New Delhi.

Kumar M., Tiwari P.S., Pande. H, \& Dadhwal V.K, 2009 A semi-automatic segmentation approach procedure for agriculture field boundary extraction in remotely sensed imagery, Indian Society of Remote Sensing symposia September 16-18 , 2009 Nagpur.

Kumar M. \& Shrivastava N. Multiresolution Segmentation And Classification Based Building Extraction Using Merged CARTOSAT And LISS IV Data, Indian Society of Remote Sensing symposia September 16-18 , 2009 Nagpur.

Kumar M., Tiwari P.S. \& Pande H., Integrating Texture Parameters in Fuzzy Rule Base Classification for Land use Land Cover Mapping, ISG Geomatics February 4- 6, 2009.

Mena J.B. 2006. Automatic vectorization of segmented road networks by geometrical and topological analysis of high resolution binary images. Elsevier B. V, pp. 704-718. Doi: 10.1016/j.knosys.2006.05.008.
Mena J. B, Malpica J. A. 2005. An automatic Method for Road Extraction in Rural and Semi-urban Areas Starting from High Resolution Satellite Imagery, Elsevier B. V, pp. 1201-1220. Doi: 10.1016/j.patrec.2004.11.005

Mohammadzadeh A., Zoej M. M. V., Tavakoli A. 2008. Automatic Main Road Extraction from High Resolution Satellite Imageries by Means of Self-Learning Fuzzy-GA Algorithm. Journal of Applied Science Vol 8 No. 19, pp. 1-8.

Mokhtarzade M., Zoej M. J V., Ebadi H. 2008. Automatic Road Extraction from High Resolution Satellite Images using Neural Networks, Texture Analysis, Fuzzy Clustering and Genetic Algorithms. The International Archives of the Photogrammetry, Remote Sensing and Spatial Information Sciences, Vol. XXXVII, Part B3b, pp. 549-556.

Mokhtarzade M., Zoej M. J. V. 2007. Road Detection from High-resolution Satellite Images using Artificial Neural Networks. International Journal of Applied Earth Observation and Geoinformation, No. 1364, pp. 32-40.

Shufelt Jefferey A. 1994. Exploiting Photogrammetric methods for building extraction in aerial images, International Archives of Photogrammetry and Remote Sensing. Vol. XXXI, part B6 Vienna 1996.

Song M., Civco D. 2004. Road Extraction Using SVM and Image Segmentation.Photogrammetric Engineering \& Remote Sensing Vol. 70, No. 12, December 2004, pp. 1365-1371.

Tiwari P.S, Pande H and Aye M.N, 2010, Exploiting IKONOS and Hyperion data fusion for automated road extraction. Geocarto International, Volume 25 No. 2 PP 123-132.

Wang Y., Li X., Zhang L., Zhang W. 2008 Automatic Road Extraction of Urban Area from High Spatial Resolution Remotely Sensed Imagery .The International Archives of the Photogrammetry, Remote Sensing and Spatial Information Sciences, Vol. XXXVII. Part B6b.

Wiedemann, C.; Heipke, C.; Mayer, H.; Jamet, O. 1998. Empirical evaluation of automatic extracted road axes. In Bowyer, K.; Philips, P. (Eds.), Empirical evaluation techniques in computer vision. IEEE Computer Society, Los Alamitos, pp.172-187. IEEE Computer Society Press, Los Alamitos, CA .

Xiaoxia S., Jixian Z., Zhengjun L. A Comparison of ObjectOriented and Pixel Based Classification using Quickbird Imagery. $\quad$ http://www.isprs.org/proceedings/XXXVI/2W25/source/A\%20COMPARISON\%20OF\%20OBJECT-

ORIENTED\%20AND\%20PIXEL-

BASED\%20CLASSIFICATION\%20APPROACHS\%20USING \%20QUICKBIRD.pdf accessed on 15.01.2010

Ziems M., Gerke M., Heipke C. 2007. Automatic Road Extraction from Remote Sensing Imagery Incorporating Prior Information and Color Segmentation. International Archives of Photogrammetry, Remote Sensing and Spatial Information Sciences, pp. 141-147. 